\title{
Latest Results on the Measurement of Reactor Antineutrino Oscillation at Daya Bay
}

\author{
Liang Zhan* \\ Institute of High Energy Physics, Beijing \\ On behalf of the Daya Bay Collaboration \\ E-mail: zhanl@ihep.ac.cn
}

The Daya Bay experiment is designed to precisely measure the reactor antineutrino oscillation utilizing eight functionally identical detectors placed at three underground experiment halls. Antineutrinos are generated from six reactor cores distributed with baselines from $360 \mathrm{~m}$ to $1900 \mathrm{~m}$. In 2012, the Daya Bay experiment observed the reactor antineutrino disappearance at short baseline with a significance of 5.2 $\sigma$. The Daya Bay experiment is continuously improving the precision of $\sin ^{2} 2 \theta_{13}$ and effective neutrino mass-squared difference $\left|\Delta m_{e e}^{2}\right|$ with growing statistics and better systematic uncertainties. In this talk, the latest results on the measurement of $\sin ^{2} 2 \theta_{13}$ and $\left|\Delta m_{e e}^{2}\right|$ is reported with the 1958-day data sample of neutron-gadolinium capture events. As an independent measurement, the progress of oscillation analysis using the neutron-hydrogen capture events is also reported.

XXXIX International Conference on High Energy Physics (ICHEP2018)

4-11 July 2018

Seoul, Korea

* Speaker. 


\section{Introduction}

The observations of neutrino oscillation have established that neutrinos have mass and that flavor eigenstates of neutrinos are mixtures of mass eigenstates. In the three-flavor neutrino framework, the neutrino mixing can be quantified using Pontecorvo-Maki-Nakagawa-Sakata (PMNS) matrix, which can be parameterized by three mixing angles $\left(\theta_{12}, \theta_{23}, \theta_{13}\right)$ and a CP phase $(\delta)$. The neutrino oscillation also depends on two independent mass-squared differences $\Delta m_{21}^{2}$ and $\Delta m_{31}^{2}$ (or $\Delta m_{32}^{2}$ ). Among the three mixing angles, the value of $\theta_{13}$ can be measured using reactor antineutrinos at a short baseline $(\sim \mathrm{km})$. The survival probability is given by

$$
P_{\bar{v}_{e} \rightarrow \bar{v}_{e}}=1-\cos ^{4} \theta_{13} \sin ^{2} 2 \theta_{12} \sin ^{2} \Delta_{21}-\sin ^{2} 2 \theta_{13} \sin ^{2} \Delta_{e e}
$$

The effective mass-squared difference, $\Delta m_{e e}^{2}$, defined $\operatorname{as}^{2} \sin _{e e} \equiv \cos ^{2} \theta_{12} \sin ^{2} \Delta_{31}+\sin ^{2} \theta_{12} \sin ^{2} \Delta_{32}$ with $\Delta_{i j}=1.267 \Delta m_{i j}^{2}\left(\mathrm{eV}^{2}\right) L(\mathrm{~m}) / E(\mathrm{MeV})$, can also be extracted from the energy-dependent oscillation of reactor antineutrinos.

The Daya Bay Reactor Neutrino Experiment was designed for precise measurement of the neutrino mixing angle $\theta_{13}$. A detailed description of Daya Bay experiment can be found in Refs. [1, 2]. Eight identically designed antineutrino detectors (ADs), with a 20-ton target mass each, are placed in three underground experimental halls (EH1 and EH2 as the near halls, and EH3 as the far hall), covering baselines from $360 \mathrm{~m}$ to $1900 \mathrm{~m}$.

In 2012, the Daya Bay experiment observed a non zero $\theta_{13}$ with a significance of $5.2 \sigma$ [3] via a rate-only measurement with 55 days of data. Other experiments obtained consistent results $[4,5,6,7]$. With a spectral analysis, the effective mass-squared difference $\left|\Delta m_{e e}^{2}\right|$ and $\sin ^{2} 2 \theta_{13}$ can be measured simultaneously. The precisions of $\sin ^{2} 2 \theta_{13}$ and $\left|\Delta m_{e e}^{2}\right|$ have been continuously improved by Daya Bay $[8,9,10,11,12]$. This talk reports the oscillation analysis using neutrongadolinium capture sample of 1958 days. As an independent measurement, the result with the neutron-hydrogen capture events is also reported. More details about the oscillation analysis are available in Ref. [12]

\section{Oscillation Analysis from Neutron Capture on Gadolinium}

A total of $\sim 3.5$ million ( $\sim 0.5$ million) IBD candidates for the near (far) halls were selected using the criteria described in [3]. The statistics were increased by $\sim 60 \%$ compared with the previous publication [11]. The ratio of expected background to IBD signal is less than $2 \%$ in all ADs. The IBD rate as well as the prompt and delayed energy spectra show consistency between side-by-side detectors.

Two key developments were carried out to improve the energy scale calibration in this analysis. At the end of 2015, a full Flash-ADC (FADC) readout system was installed in EH1-AD1, recording the PMT output waveforms with $1 \mathrm{GHz}$ and 10-bit resolution. The FADC system and the existing readout system are taking data simultaneously. The PMT readout charge was precisely measured via the waveform recorded by the FADC system. As a result, the electronics non-linearity was measured with a $0.2 \%$ precision. In January 2017 , a special calibration campaign was carried out to deploy ${ }^{60} \mathrm{Co}$ sources with various encapsulating materials. The shadowing effects of these 
enclosures were precisely determined, and the reconstructed energies of these $\gamma$-rays were measured with a $0.5 \%$ uncertainty. The energy non-linearity model was identical to the one published in Ref. [12], and the uncertainty was improved to be $\sim 0.5 \%$ from the previous $\sim 1.0 \%$ above $2 \mathrm{MeV}$.

Among the five categories of backgrounds, the ${ }^{9} \mathrm{Li} /{ }^{8} \mathrm{He}$ background dominates the uncertainty. The ${ }^{9} \mathrm{Li} /{ }^{8} \mathrm{He}$ background is produced by the cosmogenic production of ${ }^{9} \mathrm{Li}$ and ${ }^{8} \mathrm{He}$ and their subsequent $\beta$-n decays. Yields of the ${ }^{9} \mathrm{Li} /{ }^{8} \mathrm{He}$ background are estimated by fitting the distribution of time interval between the IBD candidate and the preceding muon. The yield fit is feasible only after applying a prompt energy cut to the IBD candidates to enhance the ${ }^{9} \mathrm{Li} /{ }^{8} \mathrm{He}$ background ratio in the IBD candidate. Thanks to the increased statistics, the prompt energy cut can be increased to $8 \mathrm{MeV}$ from the previous $3.5 \mathrm{MeV}$, resulting a cleaner selection of ${ }^{9} \mathrm{Li} /{ }^{8} \mathrm{He}$ background in the IBD candidates. As a result, the ${ }^{9} \mathrm{Li} /{ }^{8} \mathrm{He}$ background uncertainty was reduced from $50 \%$ to $30 \%$.

With the increased statistics and improved systematic uncertainties, the precision of the oscillation parameter measurement was improved. To evaluate the oscillation parameters, a $\chi^{2}$ is defined using the approach described as Method A in Ref. [12]. We obtained $\sin ^{2} 2 \theta_{13}=0.0856 \pm 0.0029$ and $\left|\Delta m_{e e}^{2}\right|=\left(2.522_{-0.070}^{+0.068}\right) \times 10^{-3} \mathrm{eV}^{2}$. Consistent results are obtained using Methods B or $\mathrm{C}$ in Ref. [12]. Statistic uncertainty contributes $60 \%(50 \%)$ of the total uncertainty in the precision of $\sin ^{2} 2 \theta_{13}\left(\left|\Delta m_{e e}^{2}\right|\right)$.

The reconstructed prompt energy spectrum observed in the EH3 is shown in Fig. 1 left panel. The best-fit prediction of EH3 spectrum based on the observed spectra in the two near halls and best-fit oscillation parameters is compared with the observed spectrum in the Fig. 1. The best-fit values of the $\sin ^{2} 2 \theta_{13}$ and $\left|\Delta m_{e e}^{2}\right|$, together with the $68.3 \%, 95.5 \%$ and $99.7 \%$ C.L. allowed regions are shown in Fig. 1 right panel.

\section{Oscillation Analysis from Neutron Capture on Hydrogen}

An independent measurement of $\sin ^{2} 2 \theta_{13}$ was obtained via the detection rate of IBDs tagged by neutron capture on hydrogen $(\mathrm{nH})$ [13], which is consistent with the result obtained in the neutron capture on gadolinium (nGd) analysis. The $\mathrm{nH}$ data sample has similar statistics as the nGd case due to the contribution by $\sim 15 \%$ of neutron captures in the gadolinium-doped liquid scintillator (GdLS) region and almost all of the neutron captures in the liquid scintillator (LS) region. New analysis approaches are being developed to utilize the spectral information of $\mathrm{nH}$ IBD prompt signals in the oscillation parameter analysis. The detector energy response, including the effects of energy leakage, energy non-linearity, energy resolution, are the key in the spectral analysis. The analysis on the oscillation parameters are being carried out.

\section{Summary}

New measurements of $\sin ^{2} 2 \theta_{13}$ and $\left|\Delta m_{e e}^{2}\right|$ using 1958-day data sample of neutron-gadolinium capture events are reported. We obtained $\sin ^{2} 2 \theta_{13}=0.0856 \pm 0.0029$ and $\left|\Delta m_{e e}^{2}\right|=\left(2.522_{-0.070}^{+0.068}\right) \times$ $10^{-3} \mathrm{eV}^{2}$, which is the more precise results in the measurement of $\sin ^{2} 2 \theta_{13}$. Independent measurement with the neutron-hydrogen capture events is being carried out. The Daya Bay experiment is 

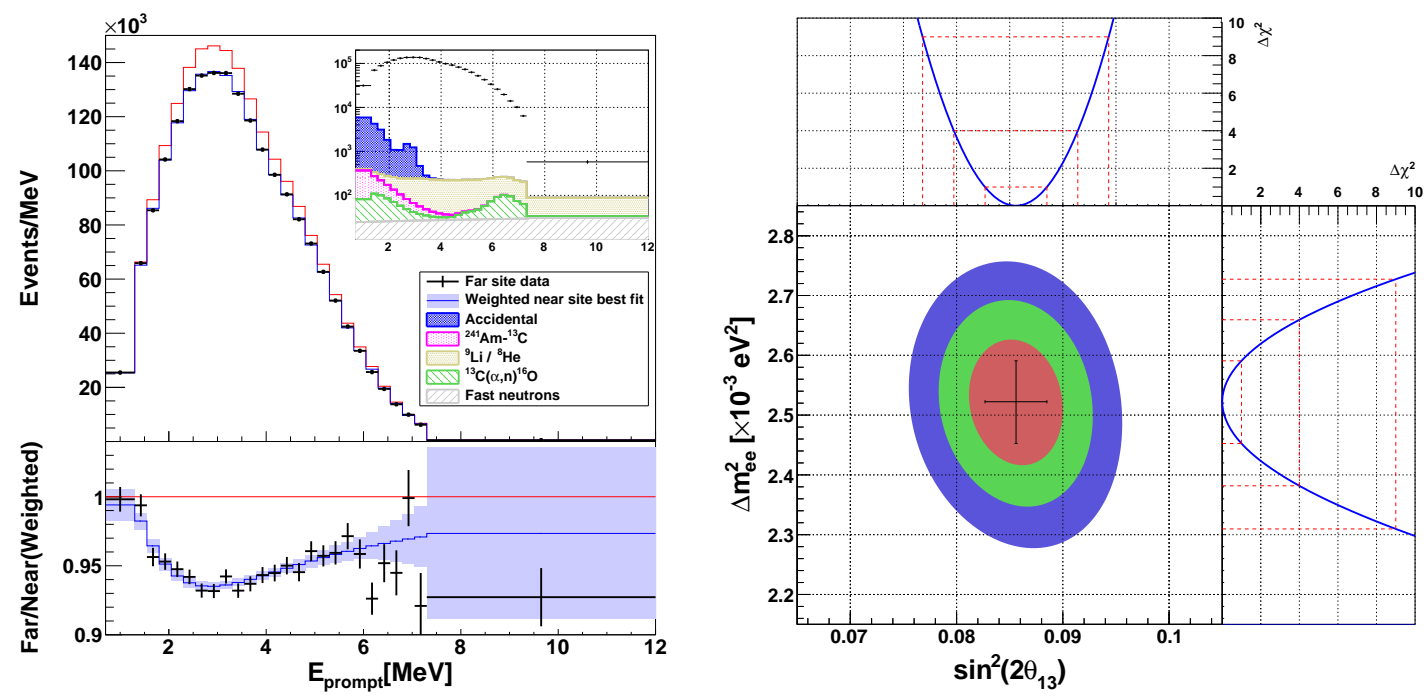

Figure 1: Left: The background-subtracted spectrum at the far site (black points) and the expectation derived from near-site measurements excluding (red line) or including (blue line) the best-fit oscillation. The bottom panel shows the ratios of data over predictions with no oscillation. The shaded area is the total uncertainty from near-site measurements and the extrapolation model. The error bars represent the statistical uncertainty of the far-site data. The inset shows the background components on a logarithmic scale. Right: The $68.3 \%$, 95.5\% and 99.7\% C.L. allowed regions in the $\sin ^{2} 2 \theta_{13}-\left|\Delta m_{e e}^{2}\right|$ plane. The one-dimensional $\chi^{2}$ for $\sin ^{2} 2 \theta_{13}$ and $\left|\Delta m_{e e}^{2}\right|$ are shown in the top and right panels, respectively. The best-fit point and one-dimensional uncertainties are given by the black cross. Figures are taken from Ref. [12].

scheduled to continue data-taking until 2020 at least, when the precision on both parameters is expected to reach $\sim 3 \%$.

\section{References}

[1] X. Guo et al. [Daya Bay Collaboration], hep-ex/0701029.

[2] F. P. An et al. [Daya Bay Collaboration], Nucl. Instrum. Meth. A 685 (2012) 78

[3] F. P. An et al. (Daya Bay collaboration), Phys. Rev. Lett. 108, 171803 (2012).

[4] K. Abe et al. (T2K collaboration), Phys. Rev. Lett. 107, 041801 (2011).

[5] P. Adamson et al. (MINOS collaboration), Phys. Rev. Lett. 107, 181802 (2011).

[6] Y. Abe et al. (Double Chooz collaboration), Phys. Rev. Lett. 108, 131801 (2012).

[7] J. K. Ahn et al. (RENO collaboration), Phys. Rev. Lett. 108, 191802 (2012).

[8] F. P. An et al. [Daya Bay Collaboration], Chin. Phys. C 37 (2013) 011001

[9] F. P. An et al. [Daya Bay Collaboration], Phys. Rev. Lett. 112, 061801 (2014)

[10] F. P. An et al. [Daya Bay Collaboration], Phys. Rev. Lett. 115 (2015) no.11, 111802

[11] F. P. An et al. [Daya Bay Collaboration], Phys. Rev. D 95 (2017) no.7, 072006

[12] D. Adey et al. [Daya Bay Collaboration], arXiv:1809.02261 [hep-ex].

[13] F. P. An et al. [Daya Bay Collaboration], Phys. Rev. D 93 (2016) no.7, 072011 